\title{
The Relationship between Brain 0scillations and BOLD Signal during Memory Formation: A Combined EEG-fMRI Study
}

\author{
Simon Hanslmayr, ${ }^{1,2,3}$ Gregor Volberg, ${ }^{1}$ Maria Wimber, ${ }^{4}$ Markus Raabe, ${ }^{1}$ Mark W. Greenlee, ${ }^{1}$ and Karl-Heinz T. Bäuml ${ }^{1}$ \\ ${ }^{1}$ Department of Experimental Psychology, University of Regensburg, D-93053 Regensburg, Germany, ${ }^{2}$ Department of Psychology and ${ }^{3}$ Zukunftskolleg, \\ University of Konstanz, D-78457 Konstanz, Germany, and ${ }^{4}$ Department of Neurology, Otto-von-Guericke University, Magdeburg, D-39120 Magdeburg, \\ Germany
}

Previous studies demonstrated that increases in the theta frequency band with concomitant decreases in the alpha/beta frequency band indicate successful memory formation. However, little is known about the brain regions and the cognitive processes that underlie these encoding-related oscillatory memory effects. We investigated this relationship using simultaneous EEG-fMRI recordings in humans during long-term memory encoding. In line with prior studies, we demonstrate that a decrease in beta power and an increase in theta power positively predict subsequent recall. In fMRI, stronger activity in the left inferior prefrontal cortex and the right parahippocampal gyrus correlated with successful memory formation. EEG source localization revealed that the subsequent memory effect in the beta band was localized in the left inferior prefrontal cortex, whereas the effect in the theta band was localized in medial temporal lobe regions. Trial-by-trial correlations between EEG and BOLD activity showed that beta power correlated negatively with left inferior prefrontal cortex activity. This correlation was more pronounced for items that could later be successfully recalled compared to items later forgotten. Based on these findings, we suggest that beta oscillations in the left inferior prefrontal cortex indicate semantic encoding processes, whereas theta oscillations in the medial temporal lobe reflect the binding of an item to its spatiotemporal context.

\section{Introduction}

Out of the stream of events we experience daily, only some events are transformed into durable memory traces. Modern neuroimaging techniques have helped to uncover the neurocognitive processes underlying successful memory formation using the subsequent memory paradigm (Paller and Wagner, 2002). In this paradigm, neural activity elicited by events that can later be retrieved is contrasted with neural activity during the encoding of events that cannot be retrieved. Numerous fMRI studies identified the left inferior frontal cortex (IFC) and the medial temporal lobe (MTL) as key regions mediating successful encoding, with higher levels of BOLD activity predicting later recall (Paller and Wagner, 2002). Different cognitive processes have been associated with these two brain regions. Whereas the left IFC is thought to reflect semantic processing (Otten and Rugg, 2001; Blumenfeld and Ranganath, 2007), the medial temporal lobe is likely involved in binding an item to its spatiotemporal context (Diana et al., 2007; Staresina and Davachi, 2009). We here demonstrate

\footnotetext{
Received Sept. 21, 2011; revised Sept. 5, 2011; accepted Sept. 14, 2011.

Author contributions:S.H., M.W., and K.-H.T.B. designed research;S.H. and G.V. performed research; M.W., M.R., and M.W.G. contributed unpublished reagents/analytic tools; S.H. and G.V. analyzed data; S.H., G.V., M.W., and K.-H.T.B. wrote the paper.

This research was supported by a grant from the Deutsche Forschungsgemeinschaft (Project HA 5622/1-1) awarded to S.H. We thank Sarang Dalal and Tzvetan Popov for helpful discussions in preparing this manuscript.

Correspondence should be addressed to Dr. Simon Hanslmayr, University of Konstanz, Department of Psychology, Box ZPR, 78457 Konstanz, Germany. E-mail: simon.hansImayr@uni-konstanz.de.

DOI:10.1523/JNEUROSCI.3140-11.2011

Copyright $\odot 2011$ the authors $\quad 0270-6474 / 11 / 3115674-07 \$ 15.00 / 0$
}

that these distinct processes are paralleled by specific changes in brain oscillatory power.

By establishing synchronous firing patterns between neural populations, brain oscillations play a critical role in shaping synaptic plasticity, and are therefore a key mechanism for successful memory encoding. Several studies using EEG/MEG recordings in humans showed that brain oscillations during encoding predict whether an event will subsequently be remembered or not (Klimesch, 1999; Düzel et al., 2010; Nyhus and Curran, 2010). These studies converge on the view that during encoding, a power increase in the theta $(4-7 \mathrm{~Hz})$ and gamma $(>25 \mathrm{~Hz})$ frequency band, together with a power decrease in the alpha/beta $(8-20 \mathrm{~Hz})$ band, predicts later recall (Klimesch et al., 1996; Weiss and Rappelsberger, 2000; Sederberg et al., 2003; Hanslmayr et al., 2009b). Theta oscillations in medial temporal lobe regions have been suggested to promote long-term memory formation via modifying synaptic plasticity (Axmacher et al., 2006; Rutishauser et al., 2010), and to reflect item-context binding (Summerfield and Mangels, 2005). Decreases in alpha and beta power have been interpreted to reflect semantic memory processes (Klimesch, 1999). Specifically, a recent study showed that power decreases in the lower beta band reflect semantic encoding (Hanslmayr et al., $2009 \mathrm{~b}$ ), suggesting a link between beta oscillations and left inferior prefrontal cortex activity. However, no study has yet successfully drawn a link between the brain oscillatory and BOLD signal subsequent memory effects (SMEs).

Examining simultaneous EEG-fMRI recordings in humans, the current study was aimed at bridging the gap between neural 
synchrony and regional BOLD activity underlying successful memory encoding. Subjects studied short lists of words and were later asked to recall as many words as possible. To integrate EEG and fMRI activity, we conducted EEG source localization as well as single-trial analyses correlating oscillatory power measures with BOLD signal. Based on previous findings, we hypothesize a functional link between beta oscillatory subsequent memory effects and BOLD signal in the left inferior prefrontal gyrus, and between theta oscillatory subsequent memory effects and BOLD signal in medial temporal lobe regions.

\section{Materials and Methods}

Subjects. Twenty-four volunteers participated in the experiment, three of them being excluded due to an insufficient number of artifact-free trials. The remaining sample (mean age: 23.1 ; range: $20-29$ years) consisted of 7 males; 20 participants were right handed. All volunteers spoke German as their native language, reported no history of neurologic or psychiatric disease, and had normal or corrected to normal vision. All participants gave their written informed consent, and the experimental protocol was approved by the local ethical review board.

Material. As study material, 240 words were drawn from the MRC Psycholinguistic Database (Coltheart, 1981), translated into German. The word material was split into 12 lists of 20 words each. The lists were matched according to word frequency (mean, 52.95; SD, 51.12), number of letters (mean, 5.36; SD, 1.15), syllables (mean, 1.69; SD, 0.54), concreteness (mean, 542.9; SD, 42.5), and imagability (mean, 563.24; SD, 32.3).

Experimental protocol. The experimental data were collected as part of a larger study that focused on the neural correlates of directed forgetting (data reported elsewhere; for a recent review on directed forgetting, see Bäuml et al., 2010). In the experiment, participants performed 12 studytest runs. Within each run, participants studied a list of 20 words. In six forget-condition runs, a cue was presented in the middle of the list instructing subjects to forget the words from the first half of the list. The second half of the list always had to be remembered. For the current study, only words that were to be remembered were used for the subsequent memory analysis. The words were presented in black font on a gray background. Each word was presented for $2.5 \mathrm{~s}$, preceded by a fixation cross with a variable duration of $1.5-2.5 \mathrm{~s}$.

After encoding, a visual feature detection task was performed as a distracter task (lasting $\sim 3 \mathrm{~min}$ ), during which subjects were presented with arrays of randomly oriented Gabor patches. Half of the arrays contained a path of 10 collinearly oriented Gabor elements, and the task was to indicate whether or not an array contained a Gabor path (Field et al., 1993). Thereafter, a free recall test of 2 min duration was conducted, during which the participants were asked to recall as many words as possible. Verbal responses were recorded using an fMRI-compatible microphone (MR confon). Scanner noise was removed from the resulting audio files using the free software package Audacity (http://audacity. sourceforge.net/). The free recall test was followed by a $30 \mathrm{~s}$ resting period, during which participants were instructed to relax.

$E E G$ - recording. The EEG was recorded from 62 channels, mounted in an elastic cap (EasyCap) and positioned according to the international 10-10 system. An MRI compatible amplifier together with the "Syncbox" device was used (Brain Products). The "Syncbox" synchronizes the clock of the EEG amplifier to the clock output of the MR scanner, which facilitates off-line removal of the MR gradient artifact. FCz was used as reference electrode, and impedances were kept below $20 \mathrm{k} \Omega$. Vertical eye movements were recorded with an additional channel placed below the left eye; the ECG was recorded by an electrode placed below the left scapula to facilitate off-line removal of cardio-ballistic artifacts in the EEG data. The signals were amplified between $0.1-100 \mathrm{~Hz}$. A notch filter at $50 \mathrm{~Hz}$ was used. The EEG data were sampled at $5 \mathrm{kHz}$.

EEG - preprocessing. Before analysis, the EEG data were corrected for the MR gradient artifact and the cardio-ballistic artifact. Both artifacts were removed using the FMRIB plug-in for EEGLAB (Delorme and Makeig, 2004; Niazy et al., 2005), running under MATLAB (The MathWorks). The MR gradient artifact was corrected using a template subtraction algorithm. The exact onset of the artifact was known from triggers delivered from the MR scanner every time a new image was acquired. The average template was then subtracted from the recorded EEG time course. For template construction, a moving average of 21 neighboring images and a linear combination of the major principal components describing the residual artifacts were used. The corrected data were then down-sampled to $500 \mathrm{~Hz}$ and high-pass filtered (using a finite impulse response, FIR, filter) at $0.5 \mathrm{~Hz}$. Residual artifacts, due to incomplete gradient artifact removal or other idiographic artifacts, were identified and removed by careful visual inspection.

The cardio-ballistic artifact, which is generated by blood pulses, was corrected using the same algorithm implemented in the FMRIB plug-in. This algorithm performs a temporal principal component analysis separately for each EEG channel. The first three components were taken as an optimal basis set for describing the artifact shape, amplitude, and scale. This set was fitted to and then subtracted from each artifact occurrence. As for the removal of the MR gradient artifacts, this was performed separately for each EEG channel.

In a last step, the cleaned EEG data were subjected to an infomax independent component analysis to correct for residual artifacts (e.g., eyeblinks, eye movements, or tonic muscle activity). The data were then segmented into epochs ranging from -2.5 to $2.5 \mathrm{~s}$ relative to word onset. Before EEG analysis, the single trials were visually inspected and rejected if they contained residual artifacts. Thereafter, on average 81.4 trials (range: 35-124) and 46.5 trials (range: 18-107) remained for the laterremembered and later-forgotten conditions, respectively.

EEG - analysis. Analysis of EEG activity was performed using selfwritten MATLAB scripts. For time-frequency analysis the EEG epochs were subjected to a Gabor transformation, which transforms a signal into a complex time-frequency signal, from which the power information can be extracted. The data were filtered in a frequency range of 4-100 Hz. The filter parameter for time-frequency resolution (gamma) was set to 1 for the lower frequency range $(4-20 \mathrm{~Hz})$, to $\pi$ for the middle frequency range $(20-45 \mathrm{~Hz}$ ), and to $2 \pi$ for the high frequency range $(55-100 \mathrm{~Hz})$. This procedure of changing the time-frequency resolution of the Gabor filter with increasing frequency (analogous to a Morlet wavelet transformation) ensures that the time-frequency characteristics of the different frequency bands are optimally picked up. Power values were calculated for each single trial, and averaged across trials within the two conditions (later remembered, later forgotten). These power values were then baseline corrected using a baseline interval of -1 to $-0.5 \mathrm{~s}$ before word onset. The resulting power values represent percentage signal change with respect to that baseline (Pfurtscheller and Aranibar, 1977).

To identify significant brain oscillatory SMEs, nonparametric randomization tests were conducted for selected time-frequency windows. These time-frequency windows were determined by averaging the power data across all channels, and calculating Wilcoxon sign-rank tests between the two conditions in each time-frequency bin. Averaging the power data across all channels was done to reduce topographical noise, similar to, e.g., the global field power approach for identifying effects of interest in multichannel arrays (Michel et al., 2004). This approach ensures that only effects that are consistent across several electrode positions reach significance. An effect was taken to be significant if it spanned at least two frequency bins $(2 \mathrm{~Hz})$ and persisted for at least $0.5 \mathrm{~s}$. Nonparametric randomization tests were then conducted for these timefrequency windows to account for multiple testing due to the $62 \mathrm{EEG}$ channels. This procedure is based on the randomization approach by Blair and Karniski (1993) and has been described in detail previously (Hanslmayr et al., 2009a,b, 2010). In short, this procedure determines the level to which a given number of significant electrodes can be expected by chance, based on permutation of the observed data.

For EEG source localization, a frequency-domain adaptive spatial filtering dynamic imaging of coherent sources (DICS) algorithm (Gross et al., 2001), as implemented in the fieldtrip software (http://fieldtrip.fcdonders.nl/), was applied. This algorithm enables DICS and constructs a spatial filter optimized for a specific location (voxel), based on the cross-spectral density matrices obtained from the data. For source localization, individual boundary element models (BEMs) were used, which were constructed from individual structural brain images. The forward model for source reconstruction was computed using the OpenMEEG plugin for fieldtrip software, on a 5 
$\mathrm{mm}$ grid of source positions covering the wholebrain compartment of the BEM (Gramfort et al., 2010). Source analysis was performed for the time-frequency windows in which significant results were obtained on the scalp level. For the beamformer algorithm, it is crucial that an equal number of trials is used in each experimental condition. To meet this requirement, the same number of trials as available in the condition with the lower trial number was randomly selected.

fMRI recording and analysis. Imaging was performed using a 3 tesla MR head scanner (Siemens Allegra). During fMRI scanning, 2226-2286 whole-brain images, consisting of 34 axial slices, were continuously acquired using an interleaved, standard $\mathrm{T} 2{ }^{*}$-weighted echo-planar imaging sequence (repetition time $\mathrm{TR}=2000 \mathrm{~ms}$; echo time $\mathrm{TE}=30 \mathrm{~ms}$; flip angle $=90^{\circ} ; 64 \times 64$ matrices; in-plane resolution: $3 \times 3 \mathrm{~mm}$; slice thickness: $3 \mathrm{~mm}$ ). High-resolution sagittal T1-weighted images were acquired after the functional scans, using a magnetization-prepared rapid gradient echo sequence $(\mathrm{TR}=2250 \mathrm{~ms} ; \mathrm{TE}=2.6 \mathrm{~ms} ; 1 \mathrm{~mm}$ isotropic voxel size) to obtain a $3 \mathrm{D}$ structural scan.

Image preprocessing and statistical analysis was performed using SPM5 (Wellcome Department of Cognitive Neurology, London, UK: www.fil.ion.ucl.ac.uk/spm), running on MATLAB. After discarding the first few images of each session, time series were corrected for differences in slice acquisition time, unwarped, and spatially realigned to the first image of the session. The average maximum movement in the $x, y$, or $z$ direction was $0.799,1.373$, or 2.552 $\mathrm{mm}$, respectively, which is below the voxel size $(3 \mathrm{~mm})$. The mean functional image was coregistered with the structural image, which was then normalized to a MNI (Montreal Neurological Institute, www.mni.mcgill.ca) template in standard stereotactic space. The resulting normalization parameters were then applied to all functional images, which were smoothed with a Gaussian kernel of $8 \mathrm{~mm}$ (FWHM).

An event-related design was used for fMRI analysis. The single-subject hemodynamic responses were modeled by convolving delta-stick functions aligned to the onset of each word with a first-order canonical hemodynamic response function (Friston et al., 1995). The resulting time series were then used as regressors in a voxelwise, fixed-effects general linear model for each subject. Two separate regressors were formed corresponding to words that were later remembered and words that were later forgotten. These onsets were associated with a parametric modulator containing the single-trial power of the frequency band of interest, averaged across those time-frequency windows and electrode sites that showed significant subsequent memory effects (Fig. 1). These power values were $z$-transformed to minimize between-subjects variance, and missing values due to artifacts were replaced by zeros (i.e., the mean power across all trials). The resulting data were high-pass filtered at $256 \mathrm{~s}$. Further regressors of no interest modeling the baseline periods, free recall periods, encoding periods of to-be-forgotten words, the distracter task, sessionspecific effects, and movement parameters determined during realignment were also included in the model. Two separate models were constructed for each frequency band (theta and beta).

For analysis of conventional fMRI subsequent memory effects, individual $t$ maps were calculated by contrasting later-remembered and later-forgotten words, using the original onsets of the words. For analysis of the correlation between EEG power and BOLD signal, individual $t$ maps were derived from contrasts corresponding to the positive and negative weights of the paramet-
$\beta$-SME $\quad \theta$-SME

(17-20 Hz; 0.0-1.25 s)

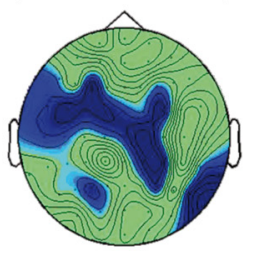

$P_{\text {corr }}<0.05$

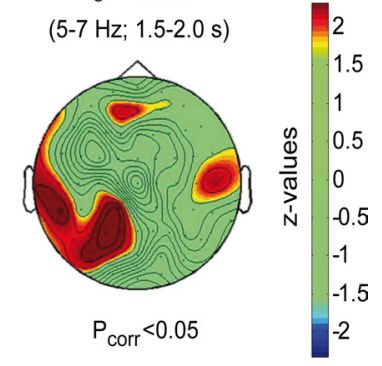

$\beta$ - Source
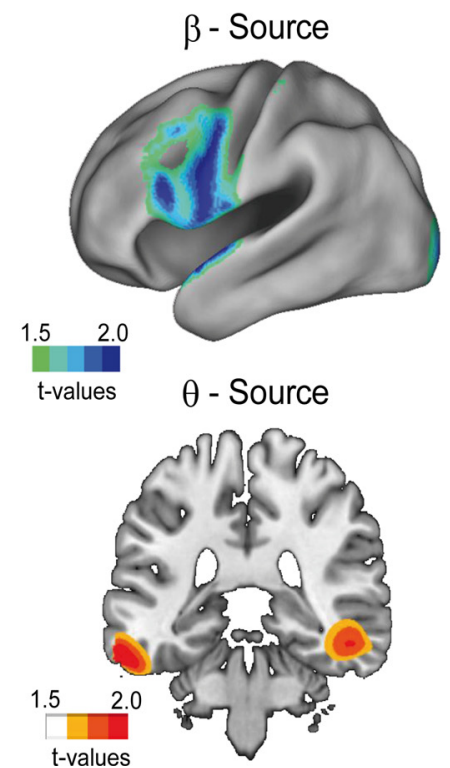

t-values

Figure 1. Brain oscillatory SMEs. $\boldsymbol{a}$, The time-frequency diagram shows the difference in oscillatory power (4-20 Hz) between later-remembered and later-forgotten items, collapsed across all 62 EEG channels. The topographies of the SMEs in the theta and shown on the right, projected onto a flattened cortical surface. c, Theta power time course for later-remembered and laterforgotten items is shown together with the source localization results on a coronal slice (right).

ric modulator. These $t$ maps were calculated separately for laterremembered and later-forgotten words. Planned comparisons were conducted for conventional subsequent memory effects, and positive and negative correlations between BOLD signal and EEG power for laterremembered words. Individual $t$ maps were then entered into a group-level, random-effects analysis. Statistical analyses were restricted to anatomically defined regions of interest (ROIs), which comprised the medial temporal lobe (bilateral hippocampus and parahippocampal cortex) and the left inferior prefrontal cortex. The WFU PickAtlas toolbox (Wake Forest University, School of Medicine, Winston-Salem; www.fmri.wfubmc.edu/cms/software) was used for ROI definition. Only clusters within these ROIs exceeding an extent threshold of 5 voxels at an uncorrected $p$ level of 0.005 are reported. An additional exploratory whole-brain analysis was conducted using a threshold of $p<0.001$ (cluster $>10$ voxels). Mean Eigenvalues of functional regions of interest were extracted using the EasyROI toolbox (www.sbirc.ed. ac.uk/cyril/cp_download.html), and BOLD signal time courses were extracted using MARSBAR (http://marsbar.sourceforge.net/).

\section{Results}

Behavioral

On average, participants recalled 64.5\% (range: $23.0-88.3 \%$ ) of the items, demonstrating that they were able to efficiently encode the word material. 
a
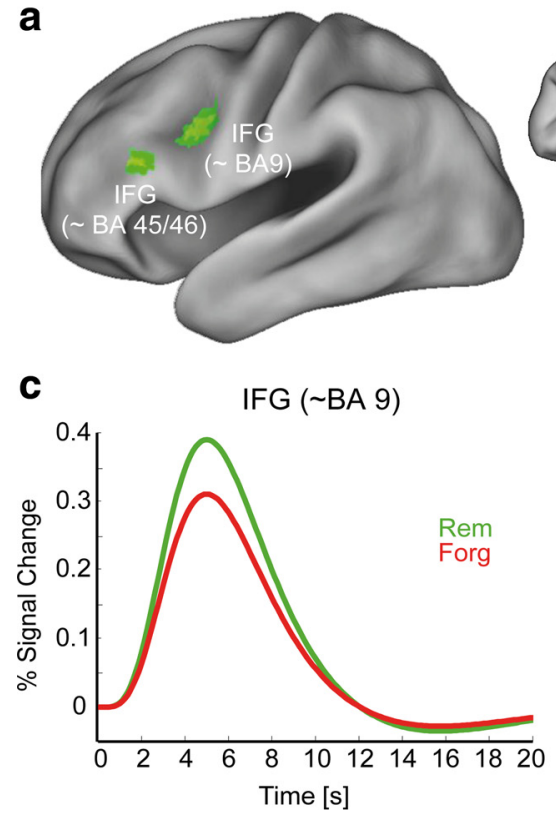
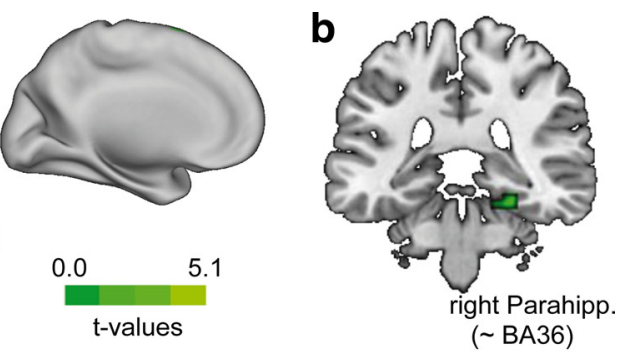

d

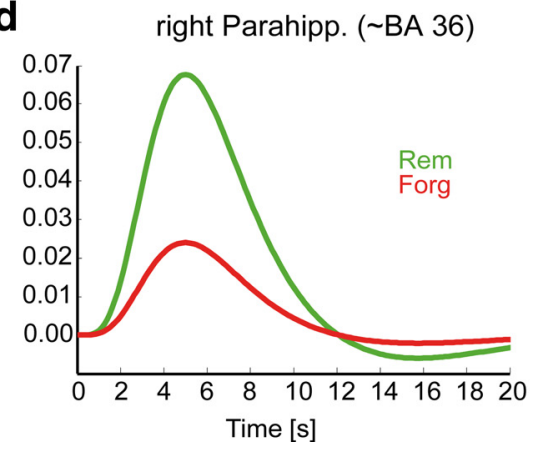

Figure 2. SMEs in the fMRI. $\boldsymbol{a}, t$ maps of voxels showing a significantly $\left(p_{\text {uncorr }}<0.005\right.$, cluster extent threshold $>5$ voxels; ROI analysis) stronger BOLD signal for later-remembered than later-forgotten words are plotted onto an inflated standard cortical surface in SPM5 space. $\boldsymbol{b}$, The subsequent memory effect in the parahippocampal gyrus is shown on a coronal slice. $\boldsymbol{c}, \boldsymbol{d}$, The time course of the BOLD signal for later-remembered (green) and later-forgotten (red) items is plotted for the left inferior frontal gyrus (c) and the right parahippocampal gyrus (d).

Table 1. Peak locations of significant fMRI subsequent memory effects (later remembered > later forgotten)

\begin{tabular}{lrllllllll}
\hline & & & & \multicolumn{7}{c}{ MNI coordinates } & \\
\cline { 6 - 7 } Anatomical label & BA & HS & Size & & $x$ & $y$ & \multicolumn{1}{c}{$z$} & $t$ \\
\hline IFG & 9 & $\mathrm{~L}$ & 76 & -48 & 9 & 30 & $5.13^{*}$ \\
MTL & 46 & $\mathrm{~L}$ & 32 & -51 & 30 & 18 & 4.31 \\
$\quad$ Parahipp. gyrus & 36 & $\mathrm{R}$ & 9 & 24 & -33 & -18 & 3.69
\end{tabular}

Cluster size is given in voxels. HS, hemisphere; L, left; $R$, right; BA, (approximate) Brodmann's area. *Survived cluster-level correction for multiple comparison $\left(p_{\text {corr }}<0.05\right)$.

\section{EEG results}

The brain oscillatory SMEs are plotted in Figure 1. Significant SMEs were obtained in the theta $(5-8 \mathrm{~Hz})$ and beta $(17-20 \mathrm{~Hz})$ frequency ranges (Fig. 1a). The SME in the beta band was evident from 0 to $1.25 \mathrm{~s}$ after stimulus onset and showed a left frontal topography. The beta oscillatory SME was due to a power decrease for later-remembered compared to later-forgotten items, and was source-localized to left inferior prefrontal regions (Fig. 1b). This effect largely replicates the SME reported in our previous study (Hanslmayr et al., 2009b). In the alpha frequency band, a similar but nonsignificant SME was observed ( $\left.p_{\text {corr }}>0.05\right)$. The SME in the theta band emerged in a later time window, from 1.5 to $2.0 \mathrm{~s}$ after stimulus onset over temporoparietal electrode sites (Fig. 1a). The theta oscillatory SME was due to higher levels of theta power for later-remembered than for later-forgotten items and was sourcelocalized to medial and ventral temporal regions (Fig. 1c). The SMEs in the theta and beta frequency band were not correlated across subjects $(p>0.5)$, and no SMEs were observed in the higher frequency ranges $(20-100 \mathrm{~Hz})$.

\section{fMRI results}

The SMEs of the fMRI are shown in Figure 2 (see Table 1 for details). The ROI analysis (see Materials and Methods) revealed

that the most pronounced SMEs emerged in the left prefrontal cortex. Two clusters were located in the left inferior frontal gyrus (IFG), covering the ventral portion of BA 9 and a more anterior region corresponding to BA 46 and extending to BA 45. A third cluster was found in the right parahippocampal cortex $(\sim \mathrm{BA} 36)$. All regions showed a more positive BOLD signal during encoding of later-recalled compared with later-forgotten items (Fig. $2 c, d)$. These results are largely consistent with previous fMRI studies (Kim, 2011). No additional regions showed SMEs in an exploratory whole-brain analysis $(p<$ 0.001 ; uncorrected; cluster $>10$ voxels).

\section{EEG-fMRI results}

Results of the trial-by-trial correlation analysis between EEG power and BOLD signal are shown in Figure 3 (see Table 2 for details). ROI analysis revealed that during encoding of later-recalled items, beta power correlated negatively with the BOLD signal in several regions within the left IFG (BA 9, BA 45/46, and BA 47), overlapping with those areas where SMEs were observed (Fig. 3a). An additional whole-brain analysis $(p<0.001$; uncorrected; cluster $>10$ voxels) revealed no further negative or positive correlations between beta power and BOLD signal. No correlations with beta power were observed during encoding of later-forgotten items. Notably, the negative correlation between beta power and BOLD signal in the two IFG regions (BA 9 and BA 45/46) was significantly more pronounced for later-recalled than for later-forgotten items $\left(F_{(1,20)}=6.04 ; p<0.05\right.$; see Fig. $\left.3 b\right)$, as revealed by a two-way ANOVA with the factors MEMORY (later remembered vs later forgotten) and REGION (BA 9 vs BA 45/46). No significant main effect for REGION or interaction was obtained ( $p$ values $>0.35$ ). No positive or negative correlations between BOLD signal and theta power were observed during encoding of later-recalled and -forgotten items in both the ROI and the whole-brain analysis.

\section{Control analysis}

As noted above (Materials and Methods), the data were collected as part of a larger study that focused on the neural correlates of directed forgetting. Although only items from the to-be-remembered conditions entered the analysis, a priori the SMEs might have been differentially affected by the instruction (remember or forget), which was given in the middle of the word lists. To account for this possibility we performed a two-way ANOVA on the fMRI data with the factors INSTRUCTION (forget vs remember) and MEMORY (later remembered vs later forgotten). Even at a very lenient threshold ( $p<0.05$; cluster $>5$ voxels), no interaction was observed in any of the regions showing SMEs. This strongly suggests that the SMEs were not differentially affected by the forget/ remember instruction.

\section{Discussion}

The results of the present study provide a first link between brain oscillatory and fMRI subsequent memory effects. Our findings indicate that beta oscillations during memory formation operate 
in the left inferior prefrontal cortex, which has consistently been linked to successful memory encoding (Wagner et al., 1998; Paller and Wagner, 2002). This conclusion is supported by converging evidence from the EEG source localization on the one hand, which identified the left inferior prefrontal cortex as the source of the beta oscillatory SME, and by the single-trial EEG-BOLD correlations on the other hand, revealing a negative correlation between beta power and BOLD signal within a left inferior prefrontal region that overlapped with the region showing a significant SME. On a more general level, this result is in accordance with previous studies reporting a negative correlation between beta power and BOLD signal in task-relevant areas (Ritter et al., 2009; Scheeringa et al., 2011).

When recording the EEG inside the MR scanner, the EEG is heavily contaminated by several artifacts (e.g., MR gradient, blood pulse) eliminated in various preprocessing steps (Niazy et al., 2005; Debener et al., 2006). To clear up potential doubts about the reliability of EEG results obtained in the MR scanner, it is crucial to validate the EEG findings based on results from similar conventional EEG/MEG studies. Indeed, the SMEs in the beta frequency band closely replicate the results from a previous EEG and a previous MEG study observing SMEs with similar topographical and temporal characteristics (Hanslmayr et al., 2009b; Meeuwissen et al., 2011). With regard to theta oscillations, our results also replicate previous studies in demonstrating that increased theta power during encoding reflects successful memory formation (Summerfield and Mangels, 2005; Osipova et al., 2006; Nyhus and Curran, 2010). The topography of the theta oscillatory SME in the current study is comparable to that obtained by Osipova et al. (2006), who also reported theta SMEs over temporal recording sites. These results suggest that reliable EEG results can be obtained during MR scanning.

In contrast to the positive SMEs (e.g., increased power for later-remembered items) that have been reported in the theta frequency band, beta oscillations typically show negative SMEs (e.g., decreased power for later remembered). This has been shown in surface (Weiss and Rappelsberger, 2000; Hanslmayr et al., 2009b) and intracranial (Sederberg et al., 2003, 2007) EEG recordings. To date, however, little is known about the functional role of beta power decreases, especially with regard to memory formation. We suggest that beta oscillations in the left inferior prefrontal cortex reflect semantic processing of to-bememorized items. This interpretation is corroborated by a prior EEG study that showed that beta oscillations during memory encoding specifically index semantic encoding (Hanslmayr et al., 2009b), and by fMRI studies that demonstrated that activity in the left inferior prefrontal cortex supports semantic processing (Gabrieli et al., 1998; Otten and Rugg, 2001). Similar to this prefrontal region, beta oscillations thus are likely to promote semantic processing of the memory material, which typically leads to rich, easily accessible memory traces (Craik and Lockhart, 1972). Importantly, a correlation between beta power and prefrontal BOLD signal was obtained only during the encoding of items that could later be recalled, but not for those items that were later forgotten. This result suggests that correlations between BOLD signal and brain oscillations depend on the current state of the neural system (e.g., whether semantic processes and their corre-
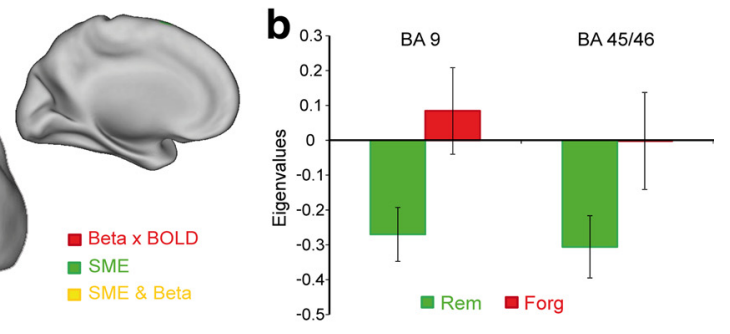

Figure 3. Single-trial correlations between beta power and BOLD signal. $\boldsymbol{a}, t$ maps of voxels showing significant $\left(p_{\text {uncorr }}<\right.$ 0.005 , cluster extent threshold $>5$ voxels; ROI analysis) negative correlations with beta power are shown during encoding of later-recalled items. Red areas correspond to brain regions showing negative correlations between beta power and BOLD; green extracted from those two IFG regions (BA 9 and BA 45/46) where the negative beta power correlations overlapped with the SMEs (yellow regions). Error bars indicate mean standard error.

Table 2. Peak locations of significant negative trial-by-trial correlations between BOLD signal and beta power for later remembered items

\begin{tabular}{lrlllrrrr}
\hline & & & & \multicolumn{7}{c}{ MNI coordinates } & \\
\cline { 5 - 6 } Anatomical label & BA & HS & Size & & $x$ & \multicolumn{1}{c}{$y$} & \multicolumn{1}{c}{$z$} & $t$ \\
\hline IFG & 9 & L & 38 & -39 & 6 & 36 & 3.81 \\
& 45 & L & 32 & -54 & 24 & 21 & 3.75 \\
& 47 & L & 10 & -48 & 21 & -9 & 4.20 \\
\hline
\end{tabular}

Cluster size is given in voxels. HS, hemisphere; L, left; $R$, right; BA, (approximate) Brodmann's area.

sponding correlates are currently engaged). This novel finding has broad implications for simultaneous EEG-fMRI studies in general, which, to our knowledge, have so far mostly investigated EEG-BOLD correlations in experimentally uncontrolled settings (e.g., resting states; Debener et al., 2006; Mantini et al., 2007; Laufs, 2008), and might thus not generalize to different taskactive conditions.

The SMEs in the theta frequency band were localized in medial temporal regions, close to the parahippocampal cortex that also showed fMRI SMEs. Theta oscillatory SMEs in the medial temporal lobe have previously been demonstrated in intracranial EEG recordings (Sederberg et al., 2003; Rutishauser et al., 2010), and have been suggested to modify synaptic plasticity (Axmacher et al., 2006; Düzel et al., 2010). Moreover, theta oscillatory and fMRI SMEs in the medial temporal lobe have been shown to promote the binding of an item to its spatiotemporal context (Summerfield and Mangels, 2005; Staresina and Davachi, 2009). Our finding of increased theta power in medial temporal regions for later-recalled items is consistent with this prior literature. An open question, however, is why we did not obtain a positive correlation between BOLD signal and theta oscillations in the medial temporal lobe during the encoding of later-remembered items. One possible reason is the low signal-to-noise ratio in this brain region, as indicated by the weak SMEs (e.g., only a cluster of 9 voxels showed a significant SME at a threshold of $p<0.005$, uncorrected). Alternatively, a previous meta-analysis showed that the SMEs in the medial temporal lobe are more pronounced for pictorial and associative memory material than for single words (Kim, 2011), and the single words used in the current study might thus not have sufficiently activated the medial temporal lobes. As shown by our results in the beta oscillatory frequency band, the single-trial correlations between BOLD signal and EEG can depend on whether the cognitive process activating a certain brain region is engaged or not. Therefore, future combined EEG- 
fMRI studies exploring the relation between MTL activity and theta oscillations should use an encoding task that strongly activates the MTL.

When conducting single-trial correlations between BOLD signal and EEG power, it is important to consider that effects in the two modalities can be driven by different physiological processes. Whereas BOLD signal primarily reflects metabolic demands and thus the number of active neurons within a population (Logothetis et al., 2001; Mukamel et al., 2005), EEG power can be modulated by two mechanisms. Specifically, a power increase can reflect either an increased number of active neurons (current dipoles) firing asynchronously, or a constant neural population firing with increased synchrony. In the latter case, band-specific EEG power can change solely as a function of synchrony, with no metabolic demands of an area (Nunez and Srinivasan, 2006). If theta power increases in the hippocampus were driven by such increases in synchrony, this would explain the absence of a correlation between theta power and BOLD signal in the MTL, despite both regions showing SMEs.

An interesting aspect of the current results is the temporal sequence of the SMEs in the beta and theta frequency band. Whereas the beta SME effect emerged in an early time window $(0-1.25 \mathrm{~s})$, the theta SME emerged later $(1.5-2 \mathrm{~s})$ around the time when the beta SME disappeared. This result further corroborates the assumption that beta and theta oscillatory SMEs are related to different cognitive processes, as they exhibit not only separable spatial but also distinct temporal characteristics. Based on our previous interpretations, this result suggests that subjects first process the semantics of an item, and subsequently recruit item-context binding mechanisms to successfully encode the material.

Our study is the first to shed light onto the relationship between BOLD signal and brain oscillations during successful memory formation. Our results strongly suggest that decreases in beta oscillatory power during encoding operate in the left inferior prefrontal cortex, presumably reflecting semantic processing of the memory material. Moreover, we found that the correlation between BOLD signal and beta power strongly depends on whether the item was later remembered or not, providing the first evidence that single-trial correlations between BOLD activity and EEG measures crucially depend on the cognitive and brain processes that are currently active.

\section{References}

Axmacher N, Mormann F, Fernández G, Elger CE, Fell J (2006) Memory formation by neuronal synchronization. Brain Res Rev 52:170-182.

Bäuml KH, Pastötter B, Hanslmayr S (2010) Binding and inhibition in episodic memory-cognitive, emotional, and neural processes. Neurosci Biobehav Rev 34:1047-1054.

Blair RC, Karniski W (1993) An alternative method for significance testing of waveform difference potentials. Psychophysiology 30:518-524.

Blumenfeld RS, Ranganath C (2007) Prefrontal cortex and long-term memory encoding: an integrative review of findings from neuropsychology and neuroimaging. Neuroscientist 13:280-291.

Coltheart M (1981) The MRC psycholinguistic database. Q J Exp Psychol 33A:497-505.

Craik FIM, Lockhart RS (1972) Levels of processing: a framework for memory research. J Verb Learn Behav 11:671-684.

Debener S, Ullsperger M, Siegel M, Engel AK (2006) Single-trial EEG-fMRI reveals the dynamics of cognitive function. Trends Cogn Sci 10:558-563.

Delorme A, Makeig S (2004) EEGLAB: an open source toolbox for analysis of single-trial EEG dynamics including independent component analysis. J Neurosci Methods 134:9-21.
Diana RA, Yonelinas AP, Ranganath C (2007) Imaging recollection and familiarity in the medial temporal lobe: a three-component model. Trends Cogn Sci 11:379-386.

Düzel E, Penny WD, Burgess N (2010) Brain oscillations and memory. Curr Opin Neurobiol 20:143-149.

Field DJ, Hayes A, Hess RF (1993) Contour integration by the human visual system: evidence for a local 'association field'. Vision Res 33:173-193.

Friston KJ, Holmes AP, Worsley KJ, Poline JB, Frith C, Frackowiak RSJ (1995) Statistical parametric maps in functional imaging: a general linear approach. Hum Brain Mapp 2:189-210.

Gabrieli JD, Poldrack RA, Desmond JE (1998) The role of left prefrontal cortex in language and memory. Proc Natl Acad Sci U S A 95:906-913.

Gramfort A, Papadopoulo T, Olivi E, Clerc M (2010) OpenMEEG: opensource software for quasi static bioelectromagnetics. Biomed Eng Online 9:45.

Gross J, Kujala J, Hamalainen M, Timmermann L, Schnitzler A, Salmelin R (2001) Dynamic imaging of coherent sources: studying neural interactions in the human brain. Proc Natl Acad Sci U S A 98:694-699.

Hanslmayr S, Leipold P, Pastötter B, Bäuml KH (2009a) Anticipatory signatures of voluntary memory suppression. J Neurosci 29:2742-2747.

Hanslmayr S, Spitzer B, Bäuml KH (2009b) Brain oscillations dissociate between semantic and nonsemantic encoding of episodic memories. Cereb Cortex 19:1631-1640.

Hanslmayr S, Staudigl T, Aslan A, Bäuml KH (2010) Theta oscillations predict the detrimental effects of memory retrieval. Cogn Affect Behav Neurosci 10:329-338.

Kim H (2011) Neural activity that predicts subsequent memory and forgetting: a meta-analysis of $74 \mathrm{fMRI}$ studies. Neuroimage 54:2446-2461.

Klimesch W (1999) EEG alpha and theta oscillations reflect cognitive and memory performance: a review and analysis. Brain Res Brain Res Rev 29:169-195.

Klimesch W, Schimke H, Doppelmayr M, Ripper B, Schwaiger J, Pfurtscheller G (1996) Event-related desynchronization (ERD) and the Dm effect: does alpha desynchronization during encoding predict later recall performance? Int J Psychophysiol 24:47-60.

Laufs H (2008) Endogenous brain oscillations and related networks detected by surface EEG-combined fMRI. Hum Brain Mapp 29:762-769.

Logothetis NK, Pauls J, Augath M, Trinath T, Oeltermann A (2001) Neurophysiological investigation of the basis of the fMRI signal. Nature 412:150-157.

Mantini D, Perrucci MG, Del Gratta C, Romani GL, Corbetta M (2007) Electrophysiological signatures of resting state networks in the human brain. Proc Natl Acad Sci U S A 104:13170-13175.

Meeuwissen E, Takashima A, Fernández G, Jensen O (2011) Evidence for human fronto-central gamma activity during long-term memory encoding of word sequences. PLoS ONE 6:e21356.

Michel CM, Murray MM, Lantz G, Gonzalez S, Spinelli L, Grave de Peralta R (2004) EEG source imaging. Clin Neurophysiol 115:2195-2222.

Mukamel R, Gelbard H, Arieli A, Hasson U, Fried I, Malach R (2005) Coupling between neuronal firing, field potentials, and fMRI in human auditory cortex. Science 309:951-954.

Niazy RK, Beckmann CF, Iannetti GD, Brady JM, Smith SM (2005) Removal of FMRI environment artifacts from EEG data using optimal basis sets. Neuroimage 28:720-737.

Nunez PL, Srinivasan R (2006) Electric fields of the brain: the neurophysics of EEG, Ed 2. New York: Oxford UP.

Nyhus E, Curran T (2010) Functional role of gamma and theta oscillations in episodic memory. Neurosci Biobehav Rev 34:1023-1035.

Osipova D, Takashima A, Oostenveld R, Fernández G, Maris E, Jensen O (2006) Theta and gamma oscillations predict encoding and retrieval of declarative memory. J Neurosci 26:7523-7531.

Otten LJ, Rugg MD (2001) Task-dependency of the neural correlates of episodic encoding as measured by fMRI. Cereb Cortex 11:1150-1160.

Paller KA, Wagner AD (2002) Observing the transformation of experience into memory. Trends Cogn Sci 6:93-102.

Pfurtscheller G, Aranibar A (1977) Event-related cortical desynchronization detected by power measurements of scalp EEG. Electroencephalogr Clin Neurophysiol 42:817-826.

Ritter P, Moosmann M, Villringer A (2009) Rolandic alpha and beta EEG rhythms' strengths are inversely related to fMRI-BOLD signal in primary somatosensory and motor cortex. Hum Brain Mapp 30:1168-1187. 
Rutishauser U, Ross IB, Mamelak AN, Schuman EM (2010) Human memory strength is predicted by theta-frequency phase-locking of single neurons. Nature 464:903-907.

Scheeringa R, Fries P, Petersson KM, Oostenveld R, Grothe I, Norris DG, Hagoort P, Bastiaansen MCM (2011) Neuronal dynamics underlying high- and low-frequency EEG oscillations contribute independently to the human BOLD signal. Neuron 69:572-583.

Sederberg PB, Kahana MJ, Howard MW, Donner EJ, Madsen JR (2003) Theta and gamma oscillations during encoding predict subsequent recall. J Neurosci 23:10809-10814.

Sederberg PB, Schulze-Bonhage A, Madsen JR, Bromfield EB, McCarthy DC, Brandt A, Tully MS, Kahana MJ (2007) Hippocampal and neocortical gamma oscillations predict memory formation in humans. Cereb Cortex 17:1190-1196.

Staresina BP, Davachi L (2009) Mind the gap: binding experiences across space and time in the human hippocampus. Neuron 63:267-276.

Summerfield C, Mangels JA (2005) Coherent theta-band EEG activity predicts item-context binding during encoding. Neuroimage 24:692-703.

Wagner AD, Schacter DL, Rotte M, Koutstaal W, Maril A, Dale AM, Rosen BR, Buckner RL (1998) Building memories: remembering and forgetting of verbal experiences as predicted by brain activity. Science 281:1188-1191.

Weiss S, Rappelsberger P (2000) Long-range EEG synchronization during word encoding correlates with successful memory performance. Brain Res Cogn Brain Res 9:299-312. 